\title{
Incentivo à ciclomobilidade em Curitiba-PR: desenvolvimento do modelo lógico dos programas Pedala Curitiba e CicloLazer
}

\author{
Incentive to cyclomobility in Curitiba-PR: Development of the logical model of the \\ Pedala Curitiba and Ciclolazer programs
}

\begin{abstract}
AUTORES
Alice Tatiane da Silva ${ }^{1,2,3}$

Adriano Akira Ferreira Hino ${ }^{2,4,5}$ (D)

Rodrigo Siqueira Reis ${ }^{1,2,6}$ (D)

1 Universidade Federal do Paraná. Programa de Pós-Graduação em Educação Física (PPGEDF) Curitiba, PR, Brasil.

2 Pontifícia Universidade Católica do Paraná. Grupo de Pesquisa em Atividade Física e Qualidade de Vida (GPAQ). Curitiba, PR, Brasil.

3 Secretaria Municipal de Esporte e Lazer (SEMEL). Departamento de Lazer. São José dos Pinhais, PR, Brasil.

4 Pontifícia Universidade Católica do Paraná. Programa de Pós-Graduação em Tecnologia em Saúde (PPGTS).Curitiba, PR, Brasil

5 Universidade Tecnológica Federal do Paraná. Programa de Pós-Graduação em Educação Física (PPGEDF).Curitiba, PR, Brasil.

6 Washington University in St Louis. Brown School. PreventionResearch Center. St Louis, MO, United States of America.

\section{CONTATO}

Alice Tatiane da Silva

silva.alice@outlook.com

Pontifícia Universidade Católica do Paraná.

Grupo de Pesquisa em Atividade Física e

Qualidade de Vida (GPAQ). Rua Imaculada Conceição, 1.155, Prado Velho, Curitiba-PR, Brasil. CEP 80215-901.

\section{DOI}

10.12820/rbafs.v.22n5p493-97

\begin{abstract}
RESUMO
O objetivo deste estudo foi desenvolver o modelo lógico dos programas Pedala Curitiba e Ciclolazer da cidade de Curitiba, PR. Foi empregada uma abordagem mista de métodos de pesquisa para obter e analisar informações sobre os programas. Foram conduzidas entrevistas com 32 profissionais, antigos e atuais, ligados aos programas de ciclomobilidade promovidos pela prefeitura, além de análise de documentos. As informações obtidas foram empregadas para estabelecer os elementos do modelo lógico, segundo o proposto pelo Centers for Disease Control and Prevention. Os programas apresentam características multissetoriais, sendo um fator importante para sua manutenção. Os modelos lógicos dos programas apontam para a necessidade de melhor alinhamento entre recursos, produtos e objetivos dos programas para dar maior conhecimento sobre o custo-efetividade do mesmo. Além disto, o alcance dos objetivos pode ser fortalecido com a ampliação e a regularidade de ações informativas junto à comunidade, o que pode auxiliar no processo de construção de apoio político aos programas.
\end{abstract}

Palavras-chave: Ciclismo; Atividades de lazer; Promoção da saúde; Avaliação de programas e projetos de saúde.

\begin{abstract}
The aim of this study was to develop a Logical Model of the Pedala Curitiba and Ciclolazer programs in the city of Curitiba, Brazil. A mixed method approach was used to obtain and analyze information about the programs. Interviews were conducted with 32 currents and past programs' employee. Additionally, a documental analysis was performed. The information obtained was used to elaborate the Logic Model elements, according to the proposed by the Centers for Disease Control and Prevention. The programs present multisectoral characteristics, which is an important factor for their maintenance. The programs' logic model shows the need for a better alignment between inputs, products and objectives to allow a better understanding of the cost-effectiveness. Furthermore, the objectives achievement could be strengthened by improving the reach and the frequency of the informational activities in the community, which also may help to build political support for the programs.
\end{abstract}

Keywords: Bicycling; Leisure activities; Health promotion; Program evaluation.

\section{cc) (i) $\Theta$

\section{Introdução}

No Brasil, as Ciclovias Recreativas estão presentes em diversas cidades, possibilitando a prática de atividades físicas, lazer, educativas e atendimentos em saúde ${ }^{1}$. Em geral, estas ações são promovidas por órgãos governamentais, como Secretarias de Esporte e Lazer e 
Departamentos de Trânsito ${ }^{1}$. Ainda que tais iniciativas estejam documentadas em alguns países da América Latina e no Brasil ${ }^{1-5}$, se desconhece descrições ou avaliações de Ciclovias Recreativas em Curitiba, Paraná.

Em Curitiba, a Secretaria Municipal de Esporte Lazer e Juventude (SMELJ) promove algumas ações de incentivo a ciclomobilidade, entre elas, os programas Pedala Curitiba e Ciclolazer, implementados nos anos de 2001 e 2013 respectivamente. No Pedala Curitiba são desenvolvidos passeios ciclísticos noturnos (20h00 às 22h00) às segundas, terças e quintas feiras, em sete Regionais Administrativas de Curitiba (RAC). Esta ação é destinada ao público que utiliza a bicicleta no lazer, esporte e transporte. Durante os eventos a Guarda Municipal (GM) e a Secretaria Municipal de Trânsito (SETRAN) controlam o fluxo de veículos, ainda há um veículo de apoio aos participantes com problemas mecânicos/físicos durante o trajeto. O Ciclolazer acontece aos domingos (09h00 às 16h00). Neste evento, a Praça Nossa Senhora de Salete (região central), ruas e ciclovias do seu entorno são temporariamente destinadas exclusivamente para atividades de lazer, recreação e ciclismo.

O desenvolvimento do Modelo Lógico destas iniciativas permitirá esclarecer de maneira sistemática as estratégias utilizadas, os recursos necessários e o efeito das intervenções na comunidade, tornando possíveis ajustes e melhorias em sua condução, basear avaliação dos mesmos e subsidiar sua replicação em diferentes comunidades. Portanto, o objetivo deste estudo foi desenvolver o Modelo Lógico dos programas Pedala Curitiba e Ciclolazer da cidade de Curitiba, Paraná.

\section{Métodos}

Entre novembro e dezembro/2016 foi conduzido um estudo transversal, utilizando métodos mistos de pesquisa $^{6}$. Os participantes do estudo qualitativo $(n=32)$ foram selecionados intencionalmente entre coordenadores e professores de Educação Física da SMELJ, que atuam ou atuaram nos programas, seguindo o método snow-ball. As entrevistas foram conduzidas individualmente, gravadas e transcritas por três pesquisadores treinados e duraram em média 43 minutos. Foram abordadas questões sobre insumos, atividades, produtos, objetivos e fatores influenciadores para o desenvolvimento dos programas ${ }^{8}$.

No mesmo período foi realizado um estudo quantitativo com levantamento documental de informações sobre os programas, sendo consultados dados em mí- dias sociais oficiais, site da Prefeitura e SMELJ e relatórios de gestão9.

\section{Análise dos dados}

Os dados coletados por meio das entrevistas e documentos foram classificados e organizados no formato de Modelo Lógico seguindo as etapas propostas pelo Manual para a Avaliação de Atividade Física ${ }^{8}$.

\section{Aspectos éticos}

A pesquisa foi aprovada pelos Comitês de Ética em Pesquisa da PUCPR sob o parecer no 1.783 .942 e da SMELJ sob o parecer $n^{\circ}$ 09/2016. Os participantes consentiram a participação na pesquisa.

\section{Resultados}

$\mathrm{Na}$ figura 1 é apresentado o modelo lógico para o Pedala Curitiba e Ciclolazer desenvolvidos no presente estudo. Segundo o relato dos entrevistados, o Pedala Curitiba é utilizado principalmente por homens (20-45 anos) com bom condicionamento físico visto a intensidade das atividades desenvolvidas, e de nível socioeconômico médio/alto devido aos equipamentos necessários para a participação. No Ciclolazer, as características do bairro onde está instalado e pelas atividades ofertadas, o programa parece atingir, basicamente, famílias que residem próximas ao local.

\section{Insumos}

\section{- Recursos humanos}

No período da pesquisa, todos os coordenadores e professores envolvidos em ambos os programas possuíam formação em Educação Física e foram alocados nos programas por voluntariedade ou por afinidade com o tema. Os programas contam com um coordenador geral comum, responsável por gerir, administrar e garantir apoio estrutural (carros de apoio, equipamentos de segurança, etc.) aos coordenadores de campo.

No Pedala Curitiba, os coordenadores de campo eram responsáveis por criar, divulgar e executar antecipadamente os percursos ciclísticos, enquanto a GM e SETRAN apoiavam a execução do evento. Os professores, voluntários, funcionários da GM, SETRAN e motoristas dos carros de apoio garantiam segurança aos participantes, fazendo a contenção frontal, lateral e fechamento do pelotão, impedindo a aproximação de veículos. Ainda, professores, voluntários e funcionários da GM/SETRAN faziam o fechamento parcial de cruzamento de vias para a passagem do pelotão. 


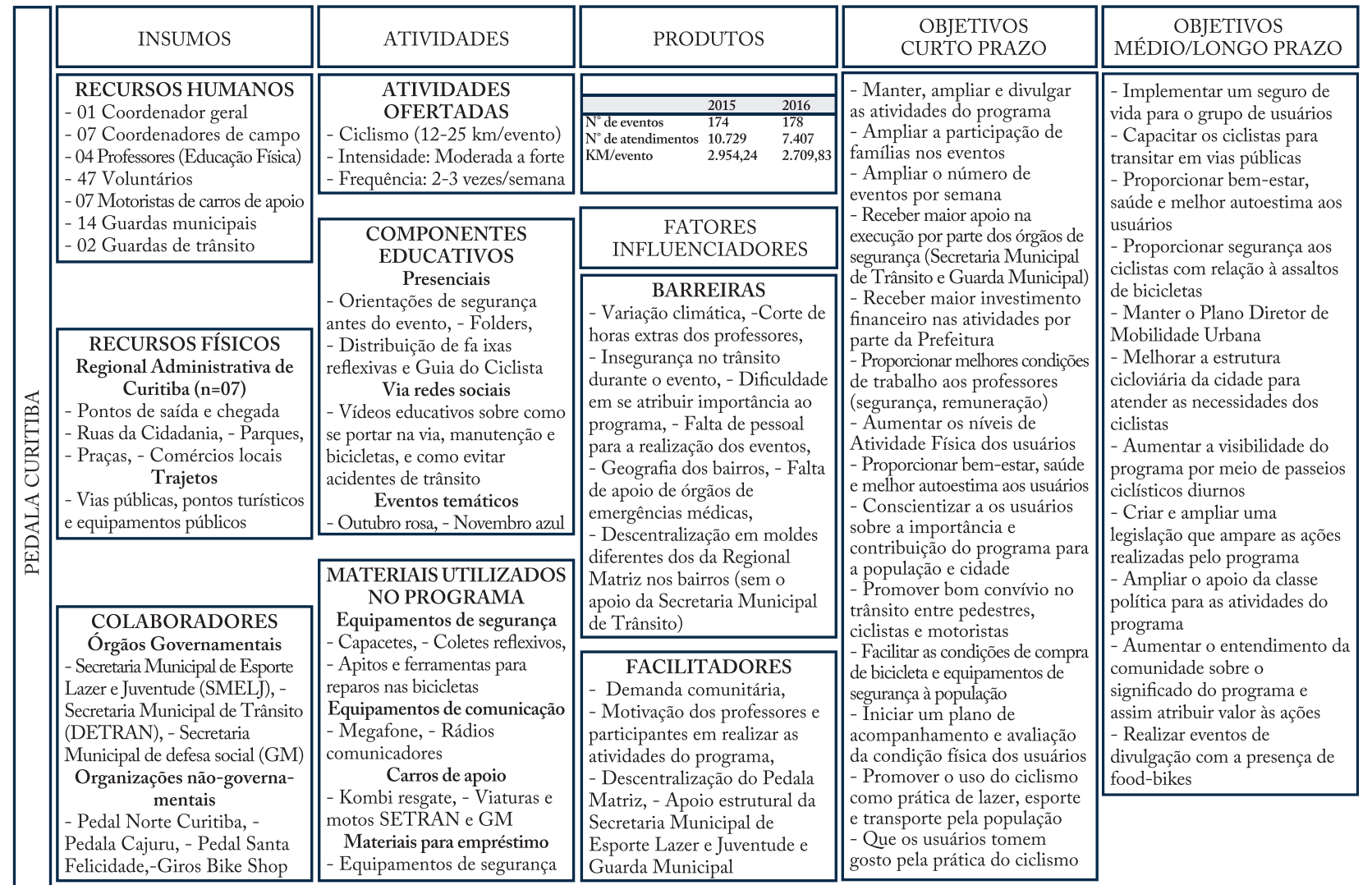

OBJETIVOS: - Fomentar o uso da bicicleta no lazer, esporte e transporte em Curitiba, - Descentralizar as atividades do Pedala Curitiba para os bairros, - Formar grupos de convivência por meio de atividades ciclísticas, - Proporcionar a prática segura do ciclismo, no período da noite

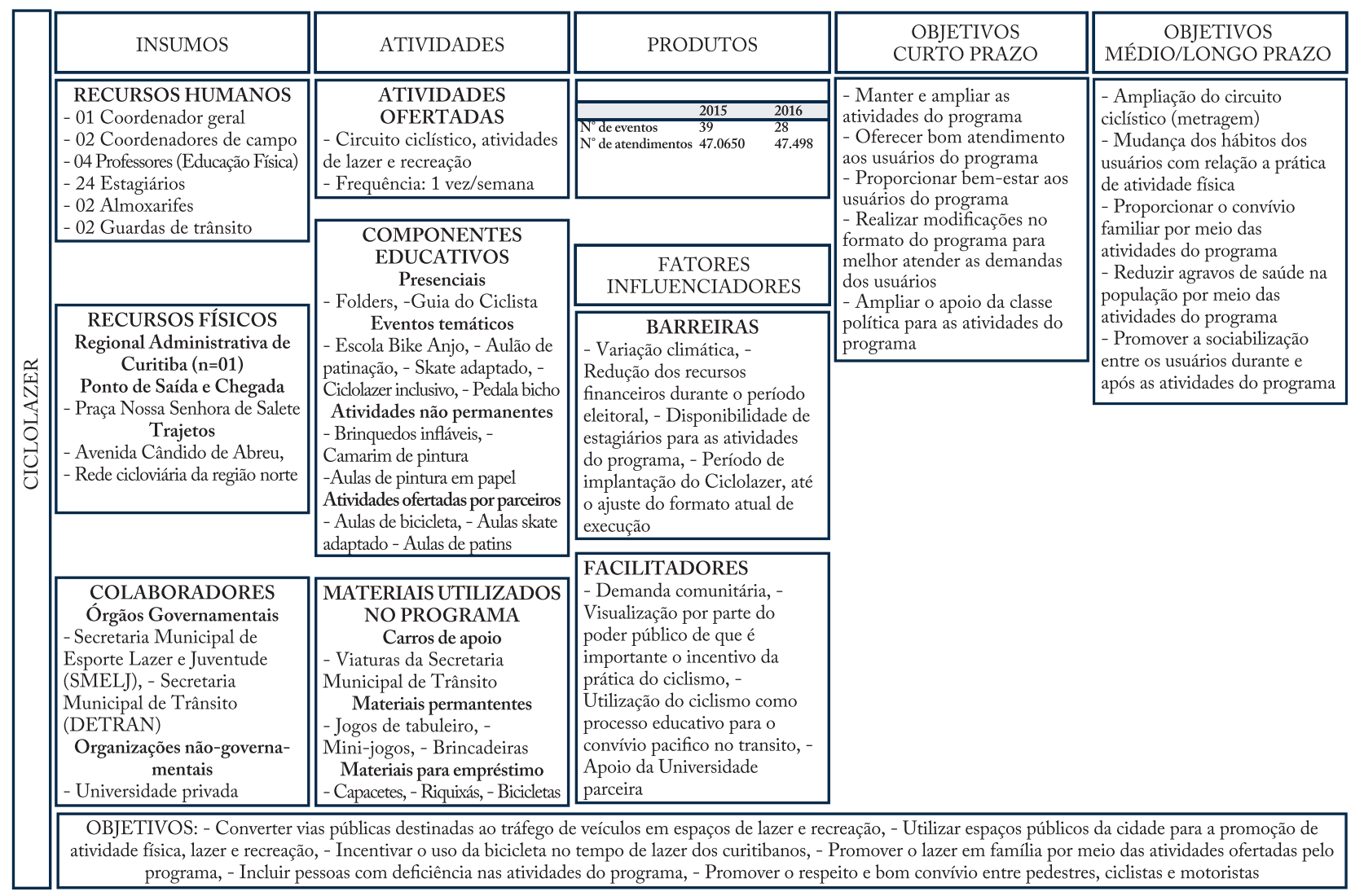

Figura 1 - Modelo lógico dos programas Pedala Curitiba e Ciclolazer desenvolvido por meio das entrevistas realizadas com profissionais (antigos e atuais) envolvidos nos programas. Curitiba-PR, 2017. 
No Ciclolazer os coordenadores de campo tinham como responsabilidade, recrutar e apoiar professores e estagiários, além de planejar, coordenar e executar o evento. Os professores realizavam a montagem do circuito ciclístico e a coordenação de atividades de lazer e recreação na praça. Os estagiários eram alunos de diversos cursos de uma universidade privada e conduziam as atividades de lazer/recreação e além de orientações do circuito ciclístico. Faziam parte do programa almoxarifes, auxiliando na separação dos materiais, e guardas de trânsito auxiliando na orientação dos veículos nos trajetos afetados pelo programa.

\section{- Recursos físicos}

O Pedala Curitiba é realizado nas ruas e ciclovias de sete $\mathrm{RAC}$, sendo estas selecionadas segundo segurança, fluxo de veículos e altimetria do terreno. O Ciclolazer é realizado exclusivamente na RAC Matriz na Praça Nossa Senhora de Salete e nas ruas e ciclovias do entorno que a integra a região norte da cidade.

\section{- Recursos financeiros}

A origem dos recursos financeiros destinados aos programas provém quase na totalidade, da Prefeitura, via orçamento anual alocado para a SMELJ. A Secretaria Municipal da Defesa Social (SMDS) e Secretaria de Trânsito (SETRAN) colaboram com o pagamento de seus profissionais e com veículos de apoio. Os estagiários do Ciclolazer recebem apoio financeiro exclusivamente da universidade de parceira. Não foram relatados ou tão pouco identificados os valores absolutos dos recursos financeiros relacionados aos programas.

\section{- Colaboradores}

O programa Pedala Curitiba é realizado por meio da parceria entre SMELJ, SETRAN e SDMS. Em algumas Regionais, a realização do programa é possível devido à parceria com grupos da comunidade e comércio local. São responsáveis pelo Ciclolazer a SMELJ, SETRAN e a Universidade parceira.

\section{- Atividades}

O ciclismo é a atividade ofertada pelo Pedala Curitiba. No Ciclolazer além do ciclismo, são ofertadas atividades de lazer/recreação. Esporadicamente são distribuídos folders educativos nas atividades do Ciclolazer, enquanto que no Pedala Curitiba as redes sociais são utilizadas para a divulgação de materiais educativos.

\section{- Produtos}

No Pedala Curitiba foram realizados 174 eventos e
10.729 atendimentos. Em 2016, foram realizados 178 eventos e 7.407 atendimentos. Com relação ao Ciclolazer, nos anos de 2015 e 2016, foram realizados 39 e 28 eventos respectivamente, totalizando quase $95 \mathrm{mil}$ atendimentos. Em 2016, sete artigos realizados pela mídia foram identificados, sendo dois para mídia televisiva, quatro para jornal (online) e um divulgado em blog.

\section{Objetivos a curto e médio prazo}

Os objetivos incluem: manter, ampliar e divulgar os programas; conscientizar os usuários sobre a importância e contribuição do programa para a população e cidade; incorporar a prática do ciclismo no dia-a-dia dos usuários.

\section{Objetivos à longo prazo}

Os objetivos incluem: manter o Plano Diretor de mobilidade urbana; criar e ampliar legislação que ampara as ações dos programas; ampliar o apoio da classe política aos programas.

\section{Fatores influenciadores}

\section{- Barreiras}

A variação climática foi a principal barreira citada para o desenvolvimento de ambos os programas. Ainda, foi citada a redução dos recursos destinados aos programas devido as eleições de 2016, com corte do pagamento de horas extras (Pedala Curitiba) e das atividades ofertadas à população (Ciclolazer), como fator negativo.

\section{- Facilitadores}

A demanda comunitária e motivação dos professores em realizar as atividades foram os aspectos positivos comuns aos dois programas. No Pedala Curitiba, a descentralização do Pedala Matriz influenciou positivamente a divulgação e procura das atividades. No Ciclolazer, o apoio financeiro e estrutural recebido da universidade parceira tornou possível a manutenção e a ampliação de atendimento.

\section{Considerações Finais}

O Modelo Lógico permitiu identificar as principais características dos programas e os respectivos desafios para a sua sustentabilidade. Os insumos para os programas envolvem diferentes setores da gestão municipal e uma instituição parceira. $\mathrm{O}$ custeio das ações, quase que na totalidade, provém do orçamento público e predominantemente de um único departamento da prefeitura. Os produtos identificados indicam o volume absoluto de atendimentos, porém não contemplam informação sobre grupos mais vulneráveis à prática de 
ciclismo. Em geral, os objetivos dos programas são parcialmente atingidos, uma vez que há limitada vinculação destes com os produtos identificados e a cobertura do programa não alcança todas regionais da cidade. Apesar de estarem presentes em diversas localidades, os programas parecem atingir apenas a parcela da população mais favorecida financeiramente e indivíduos fisicamente ativos. Os principais desafios identificados relacionam-se à manutenção dos programas, sendo baixo apoio político e apoio financeiro irregular os mais destacados. Por outro lado, a motivação tanto da equipe quanto dos participantes e a parceria com instituições privadas, foram considerados aspectos importantes para a continuidade dos programas.

As informações apontam para a necessidade de melhor alinhamento entre recursos, produtos e objetivos dos programas para dar maior conhecimento sobre o custo efetividade dos mesmos. Além disto, o alcance dos objetivos pode ser fortalecido com a ampliação e a regularidade de ações informativas junto à comunidade, o que pode auxiliar no processo de construção de apoio político aos programas.

Para a adequada interpretação dos resultados é necessário considerar que os dados apresentados são relatos dos profissionais envolvidos no desenvolvimento dos programas e que são escassos os relatórios oficiais sobre os mesmos, limitando a validação destas informações.

\section{Conflito de interesse}

Os autores declaram não haver conflitos de interesse.

\section{Financiamento}

Silva AT, recebeu bolsa da CAPES (Coordenação de Aperfeiçoamento de Pessoal de Ensino Superior) durante o Mestrado e a realização da pesquisa.

\section{Agradecimento}

Os autores agradecem os integrantes do Grupo de Pesquisa em Atividade Física e Qualidade de Vida (GPAQ/PUCPR) pela colaboração na coleta dos dados. A Secretaria Municipal de Esporte e Lazer de São José dos Pinhais (SEMEL) e ao professor Ricardo Lemes da Rosa pela contribuição no aprimoramento dos instrumentos de pesquisa. A Secretaria Municipal de Esporte, Lazer e Juventude de Curitiba (SMELJ) por disponibilizar o campo de pesquisa.

\section{Contribuição dos autores}

Silva AT, participou na concepção inicial do estudo, coleta e análise dos dados, revisão da literatura e redação do manuscrito em todas as suas etapas. Hino AAF, participou da redação e revisão crítica do manuscrito. Reis RS, foi responsável pela concepção e coordenação do projeto e participou da revisão crítica do manuscrito. Todos os autores aprovaram a versão final do manuscrito.

\section{Referências}

1. Sarmiento O, Torres A, Jacoby E, Pratt M, Schmid TL, Stierling G. The Ciclovía-Recreativa: A mass-recreational program with public health potential. J Phys Act Health. 2010;7(Suppl 2);S163-S80.

2. Movimento Conviva. Ciclovias Recreativas e Ciclofaixas de Lazer pela América Latina. Disponível em: <http:// movimentoconviva.com.br/fmb4-ciclovias-recreativas-eciclofaixas-de-lazer-pela-america-latina/>. Acesso em: 15 de janeiro de 2017.

3. Rios AP, Paez DC, Pinzón EA, Fermino RC, Sarmiento OL. Modelo lógico de la Recreovía: un programa comunitario para promoción de actividad física en Bogotá. Rev Bras Ativ Fís Saúde. 2017;22(2):206-11.

4. Sarmiento OL, Del Castillo AD, Triana CA, Acevedo MJ, Gonzalez SA, Pratt M. Reclaiming the streets for people: Insights from Ciclovías Recreativas in Latin America. Prev Med. 2017:103S:S34-S40.

5. União de Ciclistas do Brasil. Ciclovias Recreativas no Brasil. Disponível em: <http://www.uniaodeciclistas.org.br/ biblioteca/recreativas $>$ Acesso em: 23 de janeiro de 2017.

6. Turato ER. Qualitative and quantitative methods in health : Definitions, differences and research subjects. Rev Saúde Pública. 2005;39(3):507-14.

7. Curry LA, Nembhard IM, Bradley EH. Qualitative and mixed methods provide unique contributions to outcomes research. Circulation. 2009;119(20):1442-52.

8. Centers for Disease Control and Prevention. Physical Activity Evaluation Handbook. Atlanta, EUA:U.S. Department of Health and Human Services; 2002.

9. Instituto Municipal deAdministração Pública de Curitiba. Publicações do IMAP. Disponível em: < http://imap.curitiba. pr.gov.br/> Acesso em: 23 de novembro de 2017.

Recebido: 02/08/2017 Aprovado: 27/11/2017

\section{Como citar este artigo:}

Silva AT, Hino AKF, Reis RS. Incentivo à ciclomobilidade em Curitiba-PR: desenvolvimento do modelo lógico dos programas Pedala Curitiba e CicloLazer. Rev Bras Ativ Fís Saúde. 2017; 22(5):493-97. DOI: 10.12820/rbafs.v.22n5p493-97. 